\title{
Feature Extraction, Anomaly, and Change Detection on WorldView 2 Imagery by Hierarchical Image Segmentation- a study
}

Lakshman Prasad*, James Theiler, Matthew Fair, Sriram Swaminarayan

Los Alamos National Laboratory, P. O. Box 1663, Bikini Atoll Rd., Los Alamos, NM, USA 87545

\begin{abstract}
We study spatio-spectral feature extraction and image-adaptive anomaly and change detection on 8-band WorldView 2 imagery using a hierarchical polygonal image segmentation scheme. Features are represented as polygons with spectral and structural attributes, along with neighborhood structure and containment hierarchy for contextual feature identification. Further, the hierarchical segmentation provides multiple, coarse-scale, sub-backgrounds representing relatively uniform regions, which localize and simplify the spectral distribution of an image. This paves the way for facilitating anomaly and change detection when restricted to the contexts of these backgrounds. For example, forestry, urban areas, and agricultural land have very different spatio-spectral characteristics and their joint contribution to the image statistics can result in a complex distribution against which detecting anomalies could in general be a challenging problem. Our segmentation scheme provides sub-regions in the later stages of the hierarchy that correspond to homogeneous areas of an image while at the same time allowing inclusion of distinctive small features embedded in these regions. The exclusion of other image areas by focusing on these sub-backgrounds helps discover these outliers more easily with simpler methods of discrimination.

By selecting appropriate bands in WorldView2 imagery, the above approach can be used to achieve fine spatio-spectral control in searching and characterizing features, anomalies, and changes of interest. The anomalies and changes are also polygons, which have spectral and structural attributes associated with them, allowing further characterization in the larger context of the image. The segmentation and feature detections can be used as multiple layers in a Geospatial Information System (GIS) for annotating imagery.
\end{abstract}

Keywords: Image Segmentation, Feature Extraction, Change Detection, Anomaly, Hierarchy.

\section{INTRODUCTION}

Satellite image acquisition has come of age with high-resolution multiband sensor systems such as Ikonos, Quickbird, GeoEye-1, WorldView 1 and WorldView 2. With many more pixels per feature, and more spectral channels per pixel, they offer unprecedented information content. At the same time, they overwhelm analysts' ability to digest this information in a timely manner. This calls for automation of tasks such as feature extraction and change detection by computers using image analysis and machine learning. There has been a wealth of research in spectral analysis of satellite imagery ranging from subpixel signature detection to land cover classification. With today's higher resolution imagery, spatial-structural aspects of images have become more tangible and ripe for synergistic exploitation alongside multispectral information. In this paper, we describe an approach to automatically analyzing satellite imagery using a hierarchical image segmentation scheme for extracting features at multiple scales, placing features in context of their surroundings, and using the hierarchy to better pose the anomaly/change detection problem. We illustrate this on an 8band multispectral image pair captured by DigitalGlobe's WorldView 2 satellite. The images are registered and pansharpened as part of preprocessing to facilitate multispectral change detection with respect to various band combinations. This paper is organized as follows: section 2 briefly introduces a spatio-spectral hierarchical image segmentation scheme that provides a multiscale, contextual view of image content; section 3 shows how the segmentation structure can be used for spatial filtering; section 4 addresses the application of segmentation to anomaly and change detection by providing multiple contexts to selectively focus detector attention; and section 5 summarizes the paper's scope, observations, and future work.

*prasad@lanl.gov, Ph. 505-667-4587 


\section{HIERARCHICAL IMAGE SEGMENTATION}

Image segmentation is the process of decomposing an image into cohesive regions that, ideally, correspond to features in the image. A common class of approaches to segmenting images group contiguous pixels with similar spectral content into uniform regions. Many strategies have been proposed to adaptively determine similarity of pixels using local and global statistics. Even a modest summary of such approaches is beyond the scope and size of this paper. We however observe that not all features are captured by spectrally uniform regions. Indeed, even features that are spectrally uniform may appear with varying pixel values due to texture, shading, and lighting effects. Therefore, assembling regions based on spectral similarity of pixels alone may not always lead to correct separation of image features. Another approach to delineating features is to consider singularities, rather than regularities, in spectral values of image pixels. This is achieved by isolating pixels that are locations of gradient maxima. Contiguous maxima that gradually vary in strength and orientation yield coherent edges that separate features from one another. While this is a more concise description of features in terms of discriminant rather than constituent pixels, it suffers from the edges not completely or unambiguously delineating features; the edges are typically disconnected curves that fail to enclose regions. Earlier ${ }^{1}$ we have proposed a perceptually motivated region-contour hybrid scheme that obtains regional correspondences among edges to complete them into enclosing contours of feature elements. Briefly, this is achieved by computing a Delaunay triangulation of contour pixels to identify proximity relationships. Adjacent triangles are merged if their common edge does not qualify as a closure, good continuation, or transversal between contours, or a separation of distinct spectral regions represented by the triangles. Thus, retention of an edge between triangles results in a contour continuation, while deletion of an edge between triangles results in a region continuation. This dual action results in segmenting an image into polygonal feature elements. These feature elements do not always completely determine a feature, but often a part of it that is spectrally cohesive and conforms to the contours in an image. These polygonal features are further agglomerated to obtain higher-order features. Figure 1 illustrates the key stages of segmenting polygonal regions from a raster digital image comprised of pixels.

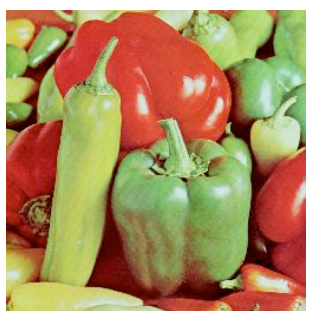

a. Digital pixel image

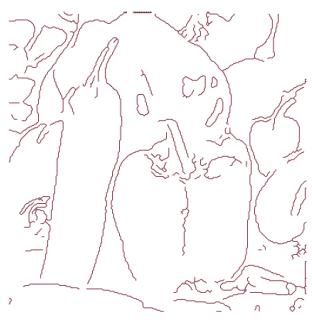

b. Gradient-based edges

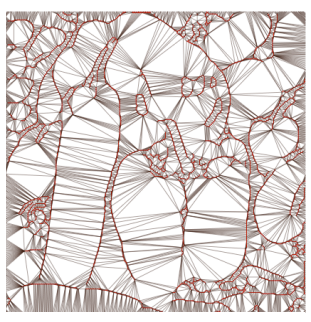

c. Delaunay tessellation

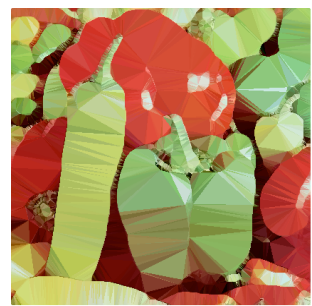

d. Spectral sampling

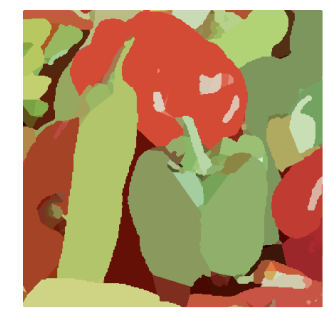

e. Polygonal grouping

Figure 1. Image segmentation by grouping of region and contour elements into polygonal features conforming to image edges

What constitutes a feature depends on what one is looking for or recognizes as meaningful. For example, an individual building is a feature, but so also is the city block in which it is found. This multiscale nature of image features cannot be captured by any single segmentation but requires a hierarchy of segmentations that progressively 'relax' the separation between features to allow the emergence of newer, larger ones comprised of smaller feature elements. We have developed $^{2}$ a hierarchical image segmentation scheme that starts with the above segmentation as a primer and merges polygons into larger ones. The merging process takes into consideration the size distribution and spectral disparity of polygonal regions as well as the structural regularity of boundaries. Ecological statistics of these parameters are computed at each level to determine the thresholds for merging based on the spatio-spectral criteria. The goal at each level is to reduce the number of polygons while maintaining spectral integrity and boundary regularity. The multiple criteria compete to establish a new assembly of polygons at the next coarser level of the hierarchical segmentation. This results in the emergence of features that tolerate some spectral variability within them-as is the case with most realworld features - while preserving salient boundaries. Figure 2 illustrates the emergence of features at multiple scales and of composite spectral content with the evolution of the hierarchy. The polygon contours at each level of the hierarchical segmentation conform to the original image gradient edges by inheriting a subset of them from their constituent 
polygons. A polygon does not intersect boundaries of polygons at other levels because of the nested inclusion property. The resulting segmentation is represented as a tree with leaf nodes consisting of the first level polygons and the root node consisting of the image rectangle. This structure helps relate features across the hierarchy and establish their spatial context in the image.
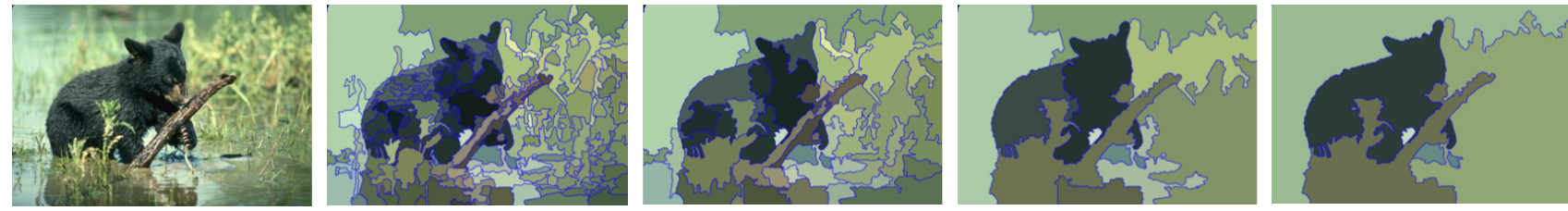

Figure 2. A digital pixel image and successively coarser levels in its hierarchical segmentation leading to discovery of new features

\section{SPATIAL FEATURE EXTRACTION}

The polygons obtained from the above method of hierarchical segmentation are described in terms of their contours and are attributed with their constituent polygons from the previous, finer level if any and with the polygons they belong to at the next coarser level if it exists. Each polygon is assigned the area-weighted average of the spectral values of its constituent polygons from the previous level. Within each level a planar graph captures the contour adjacency of polygons, with an edge between two nodes in the neighborhood graph if two polygons share a contour. The graph edges are attributed the properties of the contours they represent, such as smoothness and length, while the nodes are attributed the spectral value and area of the polygons they represent. The segmentation tree is thus equipped to efficiently address feature-related queries in terms of shape, salience, feature content, and feature context. Figure 3 illustrates a fine level segmentation (yellow contours) superposed on a portion of the WorldView 2 image used in this paper and subsequent isolation of thin shapes below a certain intrinsic aspect ratio (blue polygons and oval smooth shapes above a certain area as obtained at that level of segmentation. Subsequent levels of segmentation typically reveal other features with the same properties as polygons merge to form larger features (e.g., fragments of a road may merge into a new long feature.)
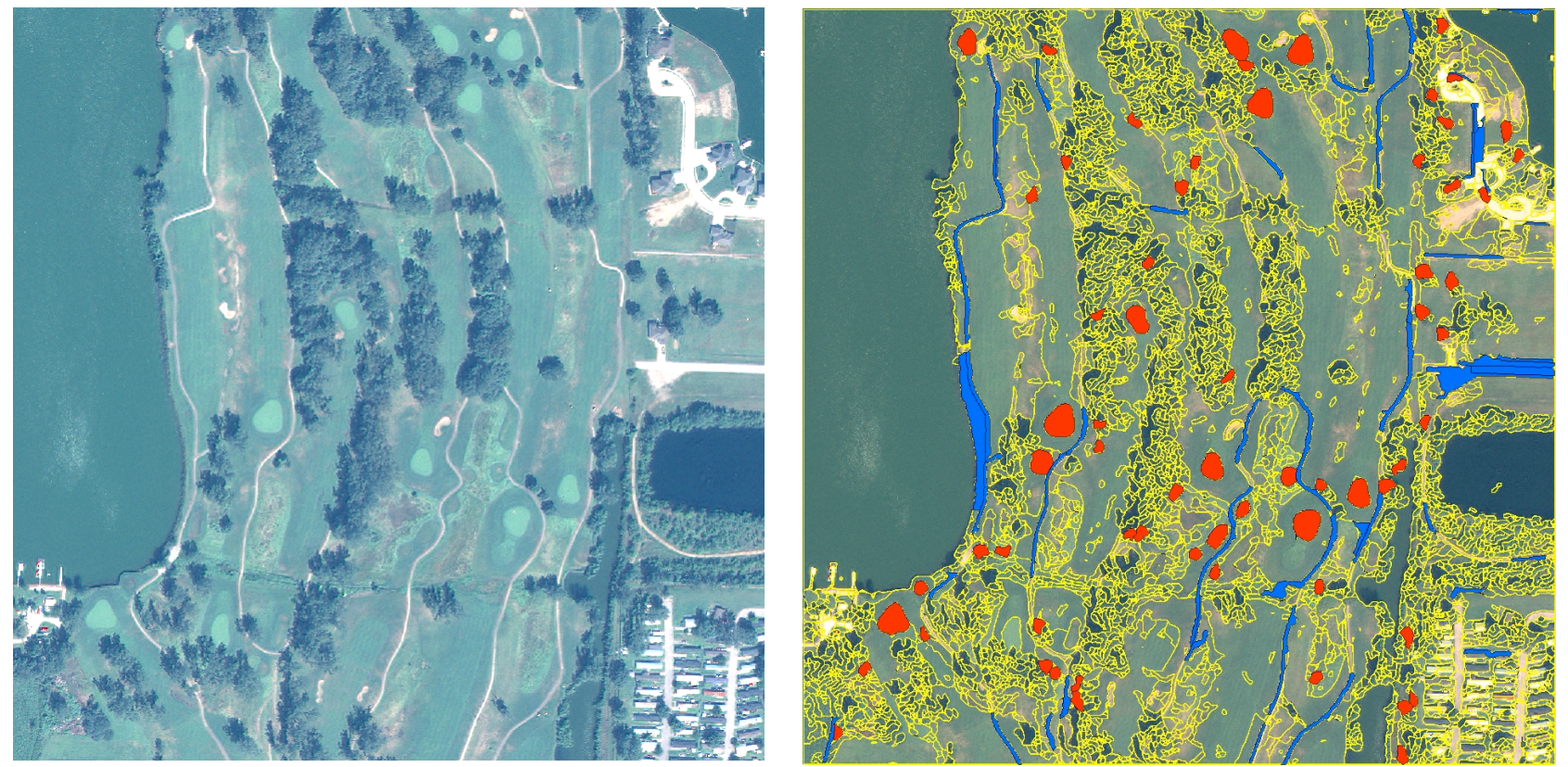

Figure 3. WorldView 2 image tile (left) and image segmentation (right) showing polygon contours in yellow and detection of polygons corresponding to long, thin features, shown in blue, and smooth oval features, shown in red. 
Both generic shape characteristics, as illustrated above, and specific shape characteristics, such as the shape of a certain building, can be sought in this framework, as well as detection of complex scenarios such as multiple co-located features of different kinds defining, say an industrial facility.

Typically, satellite images are replete with features of various kinds and it may not be practical or necessary to detect or describe all features in a large area image. Often, an analyst wants to conduct a broad area search for an unusual feature or a change in a scene. The detection of such novelty may then spawn a more thorough inquiry into the nature and category of features involved. Sifting through large quantities of imagery for the rare oddity or change is a tedious, timeconsuming, and error-prone task. Thus automating such search and detection is vital for the timely use of the vast amounts of imagery collected today.

\section{ANOMALY AND ANOMALOUS CHANGE DETECTION}

\subsection{Contextual anomaly detection}

An anomaly, by its very nature, defies definition without a reference for what is normal ${ }^{3,4}$. If an image is considered as a distribution of spectral values at various pixel locations, then outliers of this distribution may be considered anomalous. For a Gaussian distribution the Mahalanobis distance, which measures the normalized deviation of a sample point from the mean, is a good measure of anomalousness. While the Gaussian assumption is widely used in this statistical characterization of anomalousness, real-world images are not obliged to be Gaussian in their spectral distributions. Spatial contiguity of spectral uniformity as embodied by features may at best assure a mixture of Gaussians or Gaussianlike unimodal distributions to be a good approximation to image statistics. Determining outliers of a complex distribution is a hard problem in statistical machine learning ${ }^{5,6}$. All the same, anomaly detectors based on Gaussian or generalized Gaussian models have proved to be effective in many studies. In this paper, we propose that restricting such analysis to image regions that are spectrally more uniform than the whole image improves detection of subtle anomalies that would not distinguish themselves in the larger context of the whole image. Thus, a car on a farm stands out more than in a parking lot among other cars. A simple way to impose local context is to employ a moving window, and to assess the anomalousness of a pixel with respect to its local background ${ }^{7}$. However, merely restricting the spatial extent of the query region for anomalous features may not suffice, due to possible mixing of normal spectral categories within that region. We will employ an adaptive scheme that maximizes the spectral homogeneity of the query region, and thereby simplifies the local spectral distribution ${ }^{8}$.

In our hierarchical segmentation scheme, briefly described in section 2, the process of agglomeration of polygons at a certain level of the hierarchy to get the next, coarser level of segmentation is governed essentially by three broad perceptual criteria: 1) Similarity of spectral distributions of candidate merge regions, 2) size of candidate regions (i.e., small regions are encouraged to merge with their most suitable neighbors), and 3) smoothness of region boundary interfaces (i.e., regions with smooth boundaries separating them are not merged). These criteria act in conjunction to decide which regions get merged. Criterion 1 fosters spectrally homogeneous regions, while Criterion 2 fosters the extraction of larger regions. These two competing criteria result in the embedding of a few small, spectrally distinct regional features into otherwise homogeneous regions. This has the effect of providing image-adaptive, spectrally uniform backgrounds whose boundaries conform to image edges. The statistics of these backgrounds are better amenable to approximations by well-known parameterized distributions such as Gaussian or ellipsoidal functions whose outliers are easier to detect by measures such as the Mahalanobis distance. This simplification of image spectral distribution by restricting to relatively homogeneous sub backgrounds from coarser levels of the segmentation hierarchy in essence provides multiple query contexts, in each of which it is easier to detect anomalies than in the whole image taken all at once. Coarser-level polygons afford enough sample points to form a distribution and hence are preferred as backgrounds. While one can query pixels within a background, we would like to take advantage of the finest scale polygons as superpixels that not only contain structural information in terms of their boundaries conforming to image edges, but also regularize the spectral variability to avoid detection of nonstructural noisy pixels as anomalies. When anomalous polygons are detected with respect to a higher-level parent background, they can be easily grouped in the segmentation scheme to obtain shape information for characterizing the anomalies. Figure 4 illustrates this schematically, while figure 5 shows an example of contextual anomaly detection with respect to five coarse-level polygonal segments on an image 
and the distributions of spectral values of fine-scale polygons contained within them (figure $5 \mathrm{~d}$ ). The detected polygons are shown with random coloring in figure $5 \mathrm{c}$ after adjacent polygons are merged.

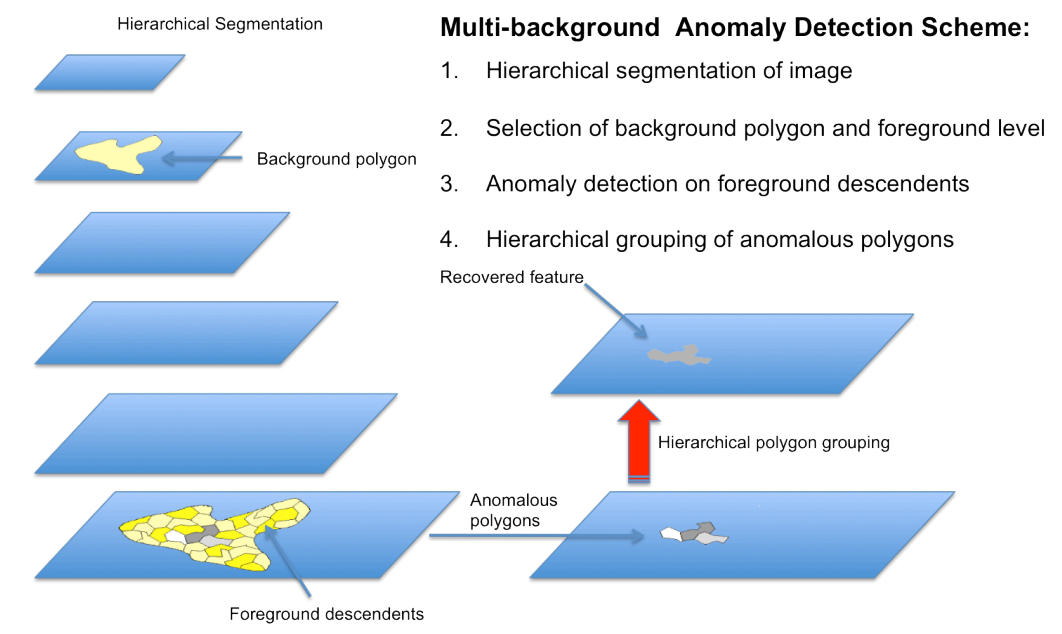

Figure 4. Schematic illustration of multibackground anomaly detection using hierarchical image segmentation

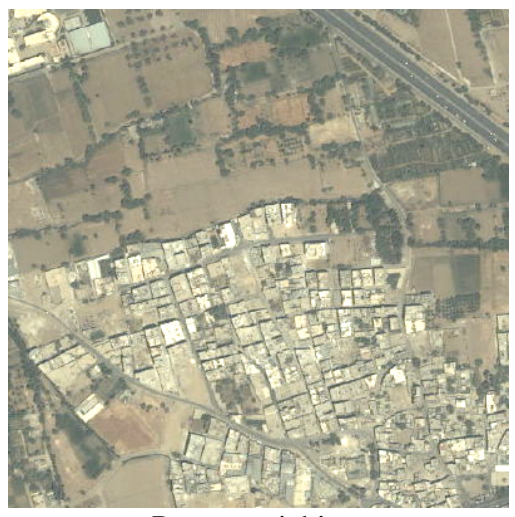

a. Raster aerial image

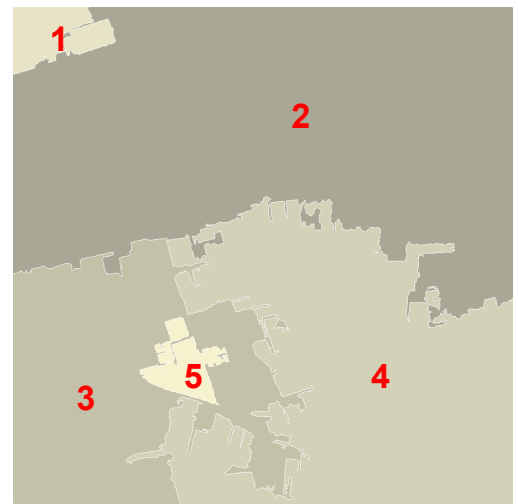

b. Coarse level background polygons

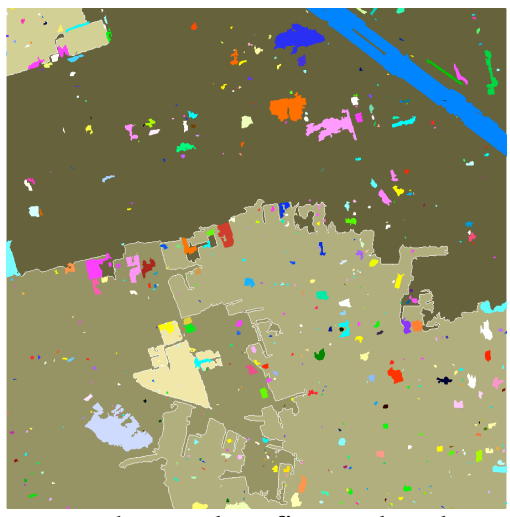

c. grouped anomalous fine-scale polygons
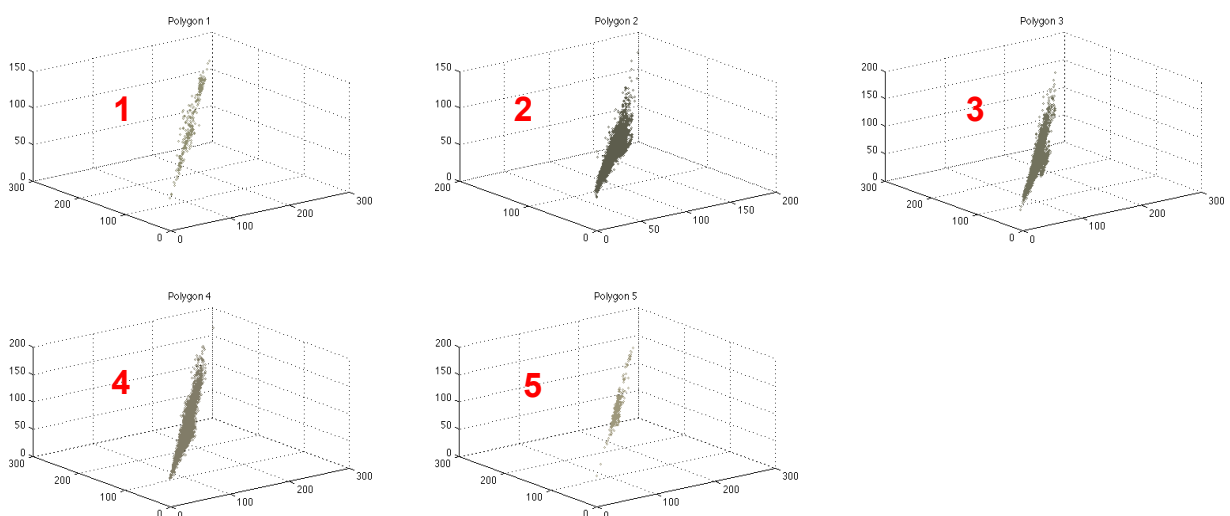

d. Distributions of RGB values of first level polygons contained in the five background polygons in $b$.

Figure 5. Illustration of background simplification for contextual anomaly detection 


\subsection{Anomalous change detection}

In detecting changes between a pair of images, one would generally like to avoid pervasive changes (such as those due to illumination or seasonal variations) while at the same time being able to detect subtle localized changes that are somehow different and perhaps therefore more interesting than the ambient variations between two images of a scene taken at two different times. To perform a pixel-to-pixel comparison the image pair would have to be co-registered so that pixels corresponding to the same scene locations have the same image location. Change detection can then be characterized as modeling the ambient variation between corresponding pixels across the image and looking for pixel pairs that differ from each other in a way that is unusual compared to how most other corresponding pixels differ. A simple way to capture the changes is to first take the pixel-wise spectral difference of the two images and consider the difference distribution. Outliers of this distribution correspond to anomalies in the difference image and are indicative of anomalous changes, as against pervasive changes, between the images. There are transformations that one could perform on the images, such as covariance equalization, ${ }^{9}$ that could mitigate the pervasive differences. It is true, however, that images can have different variabilities in different regions of the images based on the terrain and features present. Indeed, some areas are more susceptible to shadows, retention of snow, or foliage changes than others. Therefore, as in the case of anomaly detection, there are no guarantees that the difference distribution is Gaussian. Nevertheless, generalizations of Gaussians by elliptically contoured distributions have found use in radar and hyperspectral data analysis. One of the authors has proposed ${ }^{10}$ a information-theoretic, machine-learning framework for anomalous change detection between images $\boldsymbol{x}$ and $\boldsymbol{y}$, treated as random variables, by considering the likelihood ratio

$$
\frac{P(x, y)}{P(x) P(y)}
$$

where $P(\boldsymbol{x}, \boldsymbol{y})$ is a joint probability distribution over $\boldsymbol{x}$ and $\boldsymbol{y}$ that captures pervasive differences between the images, and $P(\boldsymbol{x})$ and $P(\boldsymbol{y})$ are the marginal probability distributions of the individual images. The ratio normalizes the co-occurrence of two corresponding pixel values in the two images with respect to their independent occurrence in their respective images. The likelihood ratio yields a general anomalous change detector in terms of its level curves. Thus anomalous changes correspond to low values of the ratio. When $P(\boldsymbol{x}, \boldsymbol{y})$ is Gaussian, this ratio results in the hyperbolic anomalous change detector (HACD), with a hyperbolic boundary separating pervasive and anomalous changes. ${ }^{10}$ When the joint distribution is modeled by an elliptically contoured (EC) distribution, experimental results have shown significantly better performance over a Gaussian model. ${ }^{11}$ It is reasonable to expect that when such a detector is applied to relatively homogeneous image regions obtained by image segments as described earlier, its performance should improve over when it is used without contextual specialization. In this paper we employ the EC-HACD detector ${ }^{11}$ and study its performance on a WorldView 2 image pair with and without contextual backgrounds as in the case of anomalies. The backgrounds used for restricting anomalous change detection will be obtained by hierarchically segmenting the difference image of the registered change pairs. The idea here is that the segmented regions will maximize the homogeneity of pervasive changes between the images within them and justify the Gaussian or EC approximations of the pervasive changes in these regions. As a simple example, figure 6 shows two corresponding image regions (6a, 6b) from the co-registered WV2 image pair and their absolute difference image (6c). Coarser level segments from the hierarchical segmentation of the difference image are used as backgrounds to detect outliers at a Mahalanobis distance greater than 3 from the mean of the spectral distribution of all finest level polygons contained in them (6d). Some of the changes detected are vegetative growth on the sliver of water towards the lower right of the image B, exposure of shallow bed and littoral areas of water in the upper left, absence of a pier in the lower left, change in vegetation growth and soil disturbance. Pervasive changes such as change in the color of the water body and land due to illumination are ignored. As we shall see, this simple detector, sensitized by restricting context of detection by multiple background segments, is good at picking up the strong changes, but misses some of the subtler changes that the EC-HACD detector picks up. In the absence of ground truth, we will qualitatively compare the performance of EC-HACD with and without contextual restriction from hierarchical segmentation regions. 


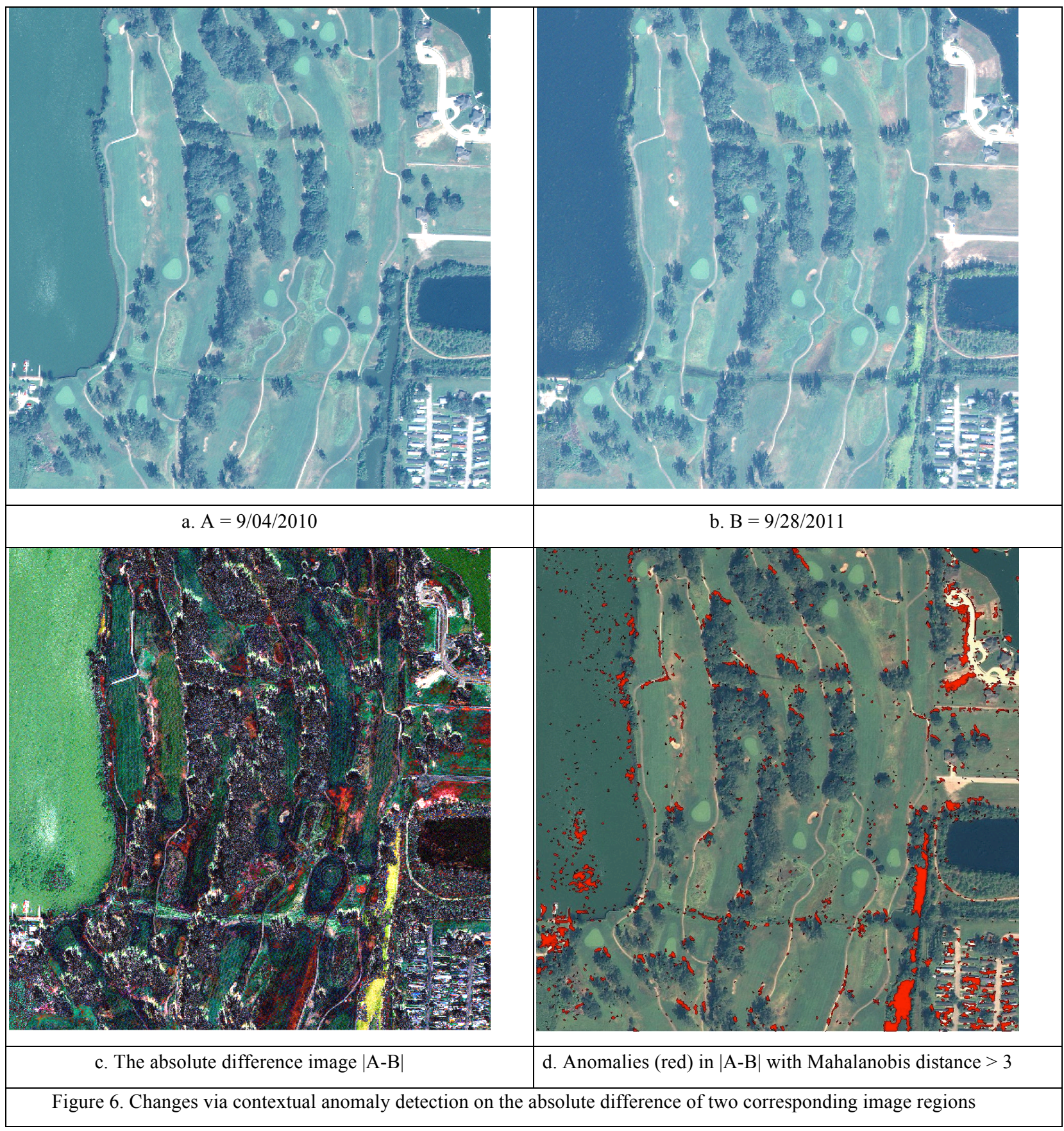

\subsection{Experiments with the contextual and non-contextual EC-HACD detector}

We will apply the EC-HACD detector with and without contextual specialization on the image pair (A, B). In both cases, instead of applying it on the pixel images, we will apply EC-HACD on two different spectral samplings of a polygonal 
decomposition of the image space. These polygons are determined by the finest level polygonal decomposition obtained from a hierarchical segmentation of the absolute difference image $|A-B|$. These polygons are spectrally sampled with respect to pixels from the images A and B to obtain two area weighted spectral distributions corresponding to A and B from a common polygonal sampling grid. This regularizes the pixel variability, conforms to the boundaries of regions of change, and allows comparison between the contextual and non-contextual change detection results. In the case of noncontextual change detection, the spectral distributions are considered in their entirety and an anomalousness score $\alpha\left(\mathrm{p}_{i}{ }^{l}\right)$ is assigned to each finest level polygon $\mathrm{p}_{i}{ }^{l}$. In the case of contextual change detection, a set of coarser segmentation levels is chosen to determine backgrounds to apply EC-HACD against. Each finest level polygon belongs to one and only one background polygon for any chosen coarse level of segmentation. Thus, with respect to its containing background polygon at that level $L$, it is assigned a score of anomalousness $\alpha_{L}\left(\mathrm{p}_{i}^{l}\right)$. The final anomalousness score of each polygon is taken to be its maximum score over all levels. By thresholding the anomalousness scores in both cases at the top $x$ percent, we get snapshots of the detector performance across common baselines. For each such threshold we can then compare the contextual and non-contextual EC-HACD results on the image pairs' sampled distributions by the ratio of the total common area of changes to the average of the total areas of changes in each image (eq. 2) as a coefficient of agreement $M$ of the two methods of applying EC-HACD. Figure 7 illustrates the changes between the two methods of applying EC-HACD at thresholded changes of top $1 \%, 5 \%$, and $10 \%$.

$$
M(A, B)=\frac{2 \sum_{u, v} \chi_{A}(u, v) \chi_{B}(u, v)}{\sum_{u, v}\left(\chi_{A}(u, v)+\chi_{B}(u, v)\right)}
$$

where $(u, v)$ indicate the pixel positions, and $\chi_{A}$ and $\chi_{B}$ are the binary indicator functions (equal to 1 if the pixel is anomalous, and zero otherwise)..

\subsection{Observations}

The degree of agreement between the two methods typically increases with the percentage of most anomalous changes considered. The exception to this trend is when a background polygon is itself a strong change and does not merge into a larger parent polygon at a coarser level for many generations. In this case, only changes within the polygon are highlighted. On the other hand the non-contextual method is free to choose any collection of polygons for candidacy of change since every polygon is in competition with every other polygon. This has the tendency of extracting the most anomalous changes first, with weaker ones showing up later in the process. In contrast, in the contextual method the background polygons shelter the subtler change polygons from stronger changes outside them and thus rank them higher than in the non-contextual case. This brings out the subtle changes at higher thresholds than in the non-contextual case. In this sense, the contextual case may be interpreted as being the more sensitive of the two methods. In the example pair, the littoral changes and shallow subsurface features are detected earlier and in greater numbers in the contextual case. On the other hand the strong vegetation changes on the surface of the sliver of water in the lower right of the images does not figure yet in the contextual case as the water body's region is a persistent background polygon that does not make all of its strongly anomalous contents available to a coarser polygon. Eventually every hierarchical segmentation terminates with the whole image rectangle as the sole polygon at the coarsest level. At this stage the results of the two methods must converge to the same with an agreement coefficient of 1 . Thus the non-contextual changes are a special case of the contextual changes when restricted to the trivial coarse level. From a practical point of view, since strong anomalous changes pop out to the analyst, the contextual method affords a less cluttered access to the harder-to-find subtle changes at higher thresholds. In our experiment, the coefficient of agreement $M$ (eq 2 ) for the thresholded changes of top $1 \%, \% 5$ and $\% 10$ were $0.5594,0.8046$, and 0.7774 , respectively. 


\section{CONCLUSIONS}

We have applied an efficient, spatio-spectral, multiscale segmentation algorithm to spatial feature extraction as well as anomalous change detection on WorldView2 imagery. The EC-HACD change detection algorithm is applied with and without spatial context to detecting changes between co-registered images of the same scene taken at different times. Our study indicates that specializing the spatial context of query for changes in an adaptive manner to conform to areas of homogeneous change patterns helps isolate subtle changes at higher anomaly scores thus reducing confusion with strong changes, which are easier to detect by a human analyst. Although we have experimented with the EC-HACD algorithm, the effect of contextual change detection would be common to other methods as well for reasons outlined in 4.4. Future work will focus on performing quantitative validations of our observations with the help of ground truth data for changes, as well as verifying the observations for a larger class of covariance-based detectors.

\section{ACKNOWLEDGMENT}

We thank DigitalGlobe Inc. for kindly providing the WorldView 2 image pair for this study. This study was partly supported by Goodrich ISR Systems, MA., and partly by the Department of Energy through a Laboratory Directed Research and Development (LDRD) grant.

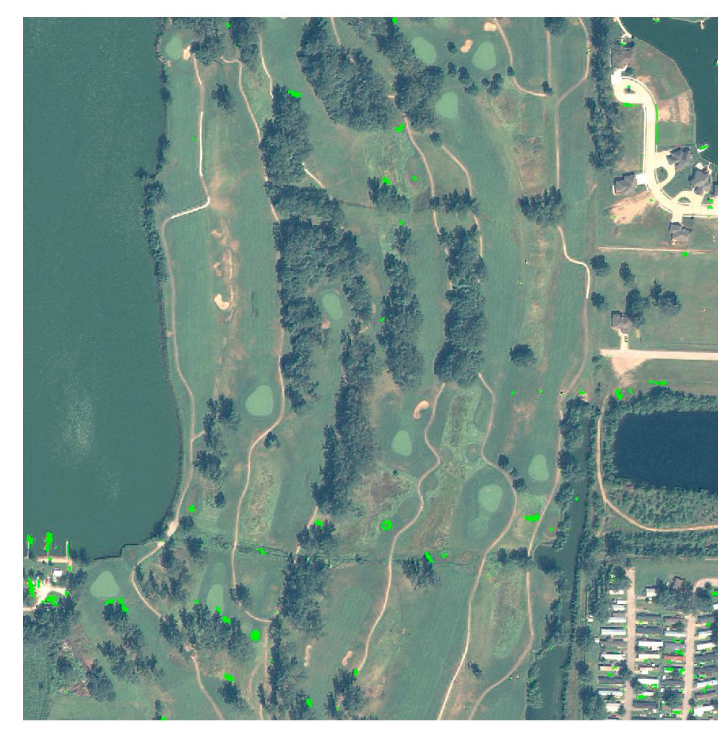

a. Top $1 \%$ non-contextual changes

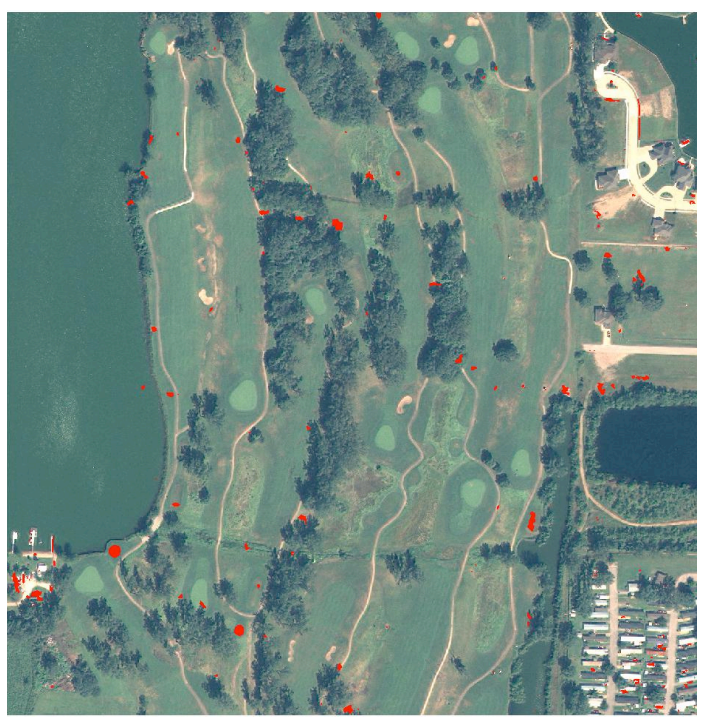

b. Top $1 \%$ contextual changes 


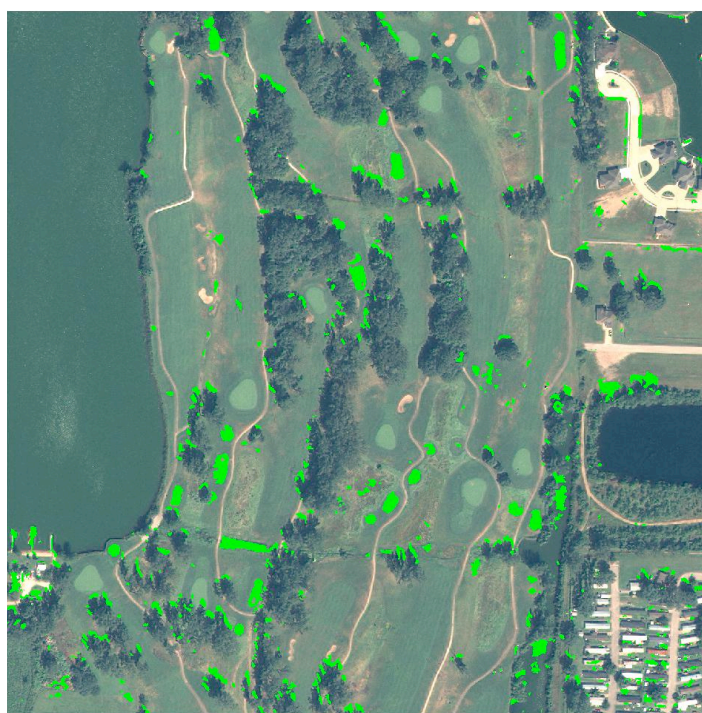

c. Top 5\% non-contextual changes

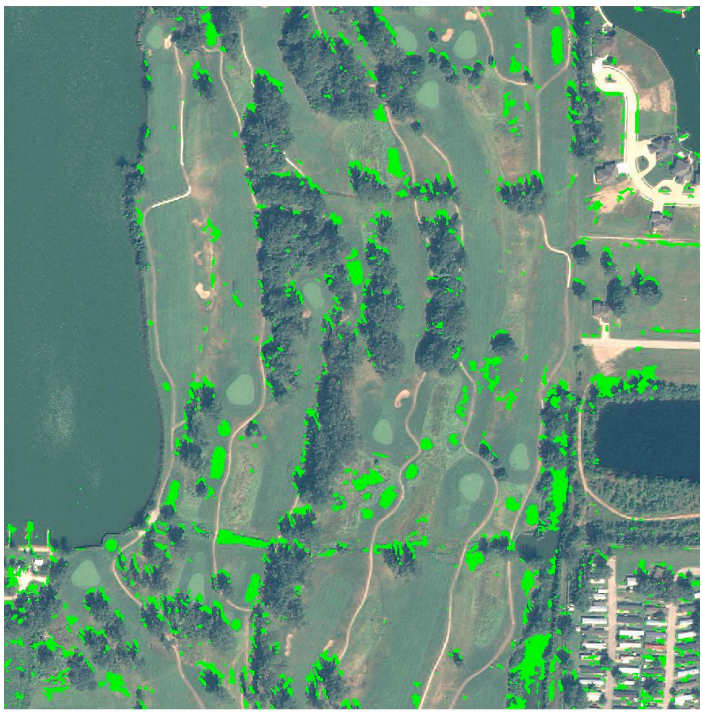

e. Top $10 \%$ non-contextual changes

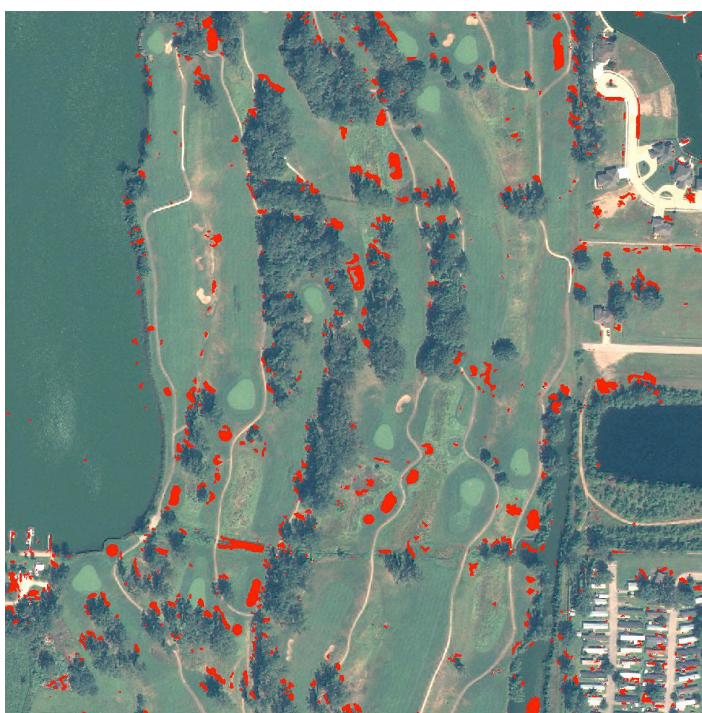

d. Top 5\% contextual changes

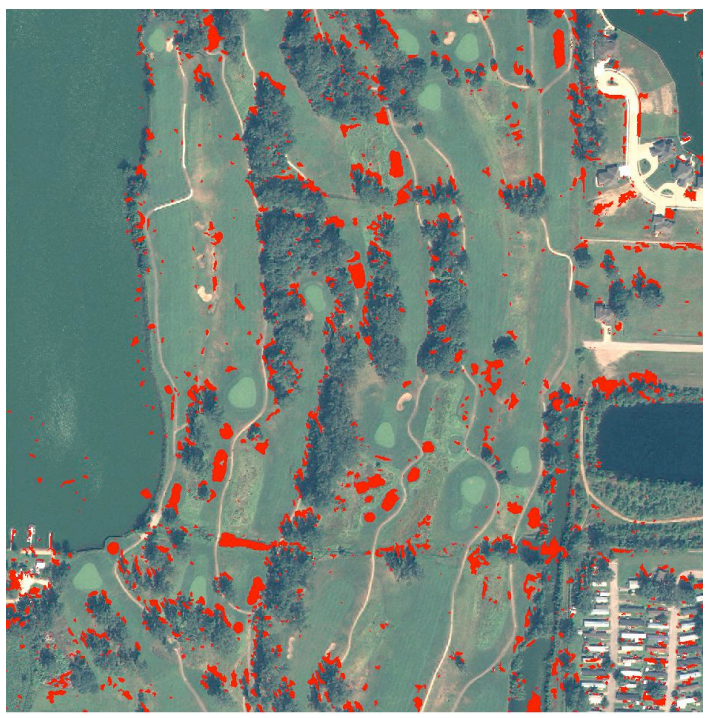

f. Top $10 \%$ contextual changes

Figure 7. Comparison of thresholded changes between non-contextual and contextual EC-HACD change detection 


\section{REFERENCES}

[1] Prasad, L. and Skourikhine, A., "Vectorized image segmentation via trixel agglomeration," Pattern Recognition, 39(4) pp 501-514 (April 2006).

[2] Prasad, L. and Swaminarayan, S., "Hierarchical Image Segmentation by Polygon Grouping," Proceedings of IEEE CVPR Workshop on Perceptual Grouping in Computer Vision, Anchorage, Alaska, (June 2008).

[3] Stein, D. W. J., Beaven, S. G., Hoff, L. E., Winter, E. M., Schaum, A. P. and Stocker, A. D., "Anomaly detection from hyperspectral imagery," IEEE Signal Processing Magazine , vol. 19, pp. 58-69 (Jan 2002).

[4] Matteoli, S., Diani, M. and Corsini, G., "A tutorial overview of anomaly detection in hyperspectral images," IEEE A\&E Systems Magazine, vol. 25, pp. 5-27 (2010).

[5] Ben-David, S. and Lindenbaum, M., "Learning distributions by their density levels: A paradigm for learning without a teacher," J. Computer and System Sciences, vol. 55, pp. 171-182 (1997).

[6] Steinwart, I., Hush, D. and Scovel, C., "A classification framework for anomaly detection," J. Machine Learning Research, vol. 6, pp. 211-232 (2005).

[7] Reed, I. S. and Yu, X., "Adaptive multiple-band CFAR detection of an optical pattern with unknown spectral distribution," IEEE Trans. Acoustics, Speech, and Signal Processing, vol. 38, pp. 1760-1770 (1990).

[8] Theiler, J. and Prasad, L., "Overlapping image segmentation for context-dependent anomaly detection," Proc. SPIE 8048, 804807 (2011).

[9] Schaum, A. and Stocker, A., "Linear chromodynamics models for hyperspectral target detection," Proc. IEEE Aerospace Conference, pp. 1879-1885 (2003).

[10] Theiler, J. and Perkins, S., "Proposed framework for anomalous change detection," ICML Workshop on Machine Learning Algorithms for Surveillance and Event Detection, pp. 7-14 (2006).

[11] Theiler, J., Scovel, C., Wohlberg, B. and Foy, B. R., "Elliptically contoured distributions for anomalous change detection in hyperspectral imagery," IEEE Geoscience and Remote Sensing Letters 7, 271-275 (2010). 